\title{
ON THE TOPOLOGICAL CENTRE OF $L^{1}(G / H)^{* *}$
}

\author{
R. RAISI TOUSI ${ }^{\otimes}$, R. A. KAMYABI-GOL and H. R. EBRAHIMI VISHKI
}

(Received 29 August 2011)

\begin{abstract}
Let $G$ be a locally compact group and $H$ be a compact subgroup of $G$. Using a general criterion established by Neufang ['A unified approach to the topological centre problem for certain Banach algebras arising in abstract harmonic analysis', Arch. Math. 82(2) (2004), 164-171], we show that the Banach algebra $L^{1}(G / H)$ is strongly Arens irregular for a large class of locally compact groups.
\end{abstract}

2010 Mathematics subject classification: primary 43A10; secondary 43A20.

Keywords and phrases: homogeneous space, topological centre, Arens product, factorisation property, Mazur property.

\section{Introduction}

In the last twenty years research on the topological centre problem has mostly centred around the Banach algebra $L^{1}(G)$, and has been dealt with by Lau et al. in the papers $[5,6,8,9]$. They showed using different approaches that $L^{1}(G)$ is strongly Arens irregular, where $G$ is a locally compact group. We recall that $A$ is said to be Arens irregular if the topological centre of $A^{* *}$ is reduced to $A$ itself. In [8] Neufang established a general criterion for a Banach algebra to be Arens irregular, which specifically led to the proof of strong Arens irregularity of the measure algebra $M(G)$ for a large class of locally compact groups.

Let $A$ be a Banach algebra and $\kappa$ be a cardinal number. We say that $A^{*}$ has the property $\left(F_{K}\right)$ if for any family of functionals $\left(h_{\alpha}\right)_{\alpha \in I} \subseteq$ Ball $\left(A^{*}\right)$ there exist a family $\left(\psi_{\alpha}\right)_{\alpha \in I} \subseteq \operatorname{Ball}\left(A^{* *}\right)$ and a single functional $h \in A^{*}$ such that the factorisation formula

$$
h_{\alpha}=h \cdot \psi_{\alpha}
$$

holds, where ' $'$ ' is the second Arens product on $A^{* *}$ and the cardinality of $I$ is at most $\kappa$.

Let $A$ be a Banach algebra and $\kappa \geq \boldsymbol{\aleph}_{0}$ be a cardinal number. A functional $f \in A^{* *}$ is called $w^{*}-\kappa$-continuous if, for all nets $\left(x_{\alpha}\right)_{\alpha \in I} \subseteq \operatorname{Ball}\left(A^{*}\right)$ of cardinality $\boldsymbol{\aleph}_{0} \leq|I| \leq \kappa$ with $x_{\alpha} \rightarrow^{w^{*}} 0$, we have $f\left(x_{\alpha}\right) \rightarrow 0$. We say that $A$ has the Mazur property of level $\kappa$ (property $\left(M_{\kappa}\right)$ ) if every $w^{*}-\kappa$-continuous functional $f \in A^{* *}$ is an element of $A$.

(c) 2012 Australian Mathematical Publishing Association Inc. 0004-9727/2012 \$16.00 
The following theorem is [8, Theorem 2.3].

Theorem 1.1. Let $A$ be a Banach algebra satisfying $\left(M_{K}\right)$ and whose dual $A^{*}$ has the property $\left(F_{\kappa}\right)$, for some $\kappa \geq \boldsymbol{\aleph}_{0}$. Then $A$ is strongly Arens irregular.

Let $G$ be a locally compact group and $H$ be a compact subgroup of $G$. Consider the homogeneous space $G / H$ with a relatively invariant measure $\mu$ which arises from a rho-function $\rho$ (see $[1,4,10])$. In [4, Theorem 4.4] it is shown that $L^{1}(G / H)$ is a Banach algebra. In this paper, for a large class of locally compact groups $G$, using Neufang's criterion (Theorem 1.1), we show that the Banach algebra $L^{1}(G / H)$ is strongly Arens irregular.

Let $G$ be a locally compact group, $H$ be a compact subgroup of $G$ and $\mu$ be a relatively invariant measure which arises from a rho-function $\rho$ on $G / H$. The mapping $T: L^{1}(G) \mapsto L^{1}(G / H)$ defined by

$$
T f(x H)=\int_{H} \frac{f(x \xi)}{\rho(x \xi)} d \xi \quad(\mu \text {-almost all } x H \in G / H)
$$

is a surjective bounded linear operator with $\|T\| \leq 1$ (see [10]). Consider $\tilde{T}$ as the mapping from $M(G)$ to $M(G / H)$ defined by

$$
\tilde{T}(\mu)(E)=\mu\left(q^{-1}(E)\right)
$$

for each Borel subset $E \subseteq G / H$ and $\mu \in M(G)$, where $q: G \rightarrow G / H$ is the canonical quotient map $q(x)=x H$. Then it is easy to see that $\tilde{T}$ is onto and $M(G / H)$ is a Banach algebra endowed with the following convolution: for $v, \hat{v} \in M(G / H)$,

$$
v * \dot{v}:=\lambda * \dot{\lambda}\left(q^{-1}(E)\right),
$$

where $\lambda, \hat{\lambda} \in M(G)$ and $\tilde{T}(\lambda)=v, \tilde{T}(\hat{\lambda})=\dot{v}($ see $[10])$.

Equip $L^{1}(G / H)^{* *}$ with the second Arens product denoted by ' ' as follows: for $m, n \in L^{1}(G / H)^{* *}, \eta \in L^{1}(G / H)^{*}, \varphi, \gamma \in L^{1}(G / H)$,

$$
\begin{aligned}
& \langle m \cdot n, \eta\rangle=\langle n, \eta \cdot m\rangle \\
& \langle\eta \cdot m, \gamma\rangle=\langle m, \gamma \cdot \eta\rangle \\
& \langle\gamma \cdot \eta, \varphi\rangle=\langle\eta, \varphi * \gamma\rangle,
\end{aligned}
$$

where ' $*$ ' is the convolution of $L^{1}(G / H)$ (see [4]).

\section{Main result}

Throughout this paper we assume that $G$ is a locally compact group and $H$ is a compact subgroup of $G$. Denote by $\kappa(G)$ and $b(G)$ the compact covering number of $G$ and the least cardinality of an open basis at the neutral element of $G$, respectively (see [8]). We show that $L^{1}(G / H)^{*}$ has the factorisation property of level $\kappa(G)$ and $L^{1}(G / H)$ satisfies the Mazur property of level $\kappa(G)$, for a large class of locally compact groups $G$. Theorem 1.1 will then imply that in this case $L^{1}(G / H)$ is strongly Arens irregular. Indeed, the main result of this paper is the following theorem. 
THEOREM 2.1. Let $G$ be a locally compact noncompact group and $H$ be a compact subgroup of $G$. Assume that $\kappa(G) \geq 2^{b(G)}$. Then $L^{1}(G / H)^{*}$ has the property $\left(F_{\kappa(G)}\right)$ and $L^{1}(G / H)$ satisfies $\left(M_{\kappa(G)}\right)$. In particular, $L^{1}(G / H)$ is strongly Arens irregular.

To prove Theorem 2.1, we first discuss the factorisation property. To begin, we establish the following lemmata. Denote by $L_{y}$ the left translation operator, defined by $L_{y} \gamma(x H)=\gamma\left(y^{-1} x H\right), x, y \in G($ see $[4])$.

Consider

$$
\delta_{y H}(x H)= \begin{cases}1 & x H=y H \\ 0 & x H \neq y H\end{cases}
$$

Denote by $\hat{\delta}_{y H}$ the image of $\delta_{y H}$ under the canonical mapping ${ }^{\wedge}: M(G / H) \rightarrow$ $M(G / H)^{* *}$.

Lemma 2.2. Let $G$ be a locally compact group and $H$ be a compact subgroup of $G$. Consider $G / H$ as the homogeneous space with relatively invariant measure $\mu$ which arises from a rho-function $\rho$. Then for $\gamma \in L^{1}(G / H)^{*}$,

$$
\gamma \cdot \hat{\delta}_{y H}=\frac{\rho(y)}{\rho(e)} L_{y^{-1}} \gamma
$$

where $e$ is the identity element of $G, y \in G$.

Proof. Let $\gamma \in L^{1}(G / H)^{*}, \eta \in L^{1}(G / H)$. Then

$$
\begin{aligned}
\left\langle L_{y^{-1}} \gamma, \eta\right\rangle & =\int_{G / H} L_{y^{-1}} \gamma(x H) \eta(x H) d \mu(x H) \\
& =\int_{G / H} \gamma(y x H) \eta(x H) d \mu(x H) \\
& =\int_{G / H} \gamma(x H) L_{y} \eta(x H) \frac{\rho\left(y^{-1}\right)}{\rho(e)} d \mu(x H) \\
& =\int_{G / H} \gamma(x H) L_{y} \eta(x H) \frac{\rho(e)}{\rho(y)} d \mu(x H),
\end{aligned}
$$

where the last equality follows from the identity (see [4])

$$
\rho(x y)=\frac{\rho(x) \rho(y)}{\rho(e)} .
$$

Therefore,

$$
\frac{\rho(y)}{\rho(e)}\left\langle L_{y^{-1}} \gamma, \eta\right\rangle=\int_{G / H} \gamma(x H) L_{y} \eta(x H) d \mu(x H) .
$$

On the other hand,

$$
\begin{aligned}
\left\langle\gamma \cdot \hat{\delta}_{y H}, \eta\right\rangle & =\left\langle\hat{\delta}_{y H}, \eta \gamma\right\rangle \\
& =\left\langle\eta \gamma, \delta_{y H}\right\rangle \\
& =\left\langle\gamma, \delta_{y H} \cdot \eta\right\rangle \\
& =\gamma\left(\delta_{y H} * \eta\right),
\end{aligned}
$$


where ' $*$ ' in the last equality is the convolution in $M(G / H)$ defined as in (1.3). To continue the calculations in (2.3), note that since $L^{1}(G / H)$ is an ideal of $M(G / H)$, $\delta_{y H} * \eta \in L^{1}(G / H)$. It is easy to see that

$$
\delta_{y H}=\tilde{T}\left(\delta_{y}\right),
$$

where $\tilde{T}$ is as in (1.2). Now choose $g \in L^{1}(G)$ such that $\eta=T g$. Then

$$
\begin{aligned}
\delta_{y H} * \eta & =\tilde{T}\left(\delta_{y}\right) * \tilde{T}(g) \\
& =\tilde{T}\left(\delta_{y} * g\right) \\
& =T\left(\delta_{y} * g\right) \\
& =\int_{H} \frac{\delta_{y} * g(x \xi)}{\rho(x \xi)} d \xi \\
& =\int_{H} \int_{G} \frac{g\left(z^{-1} x \xi\right) d \delta_{y}(z)}{\rho(x \xi)} d \xi \\
& =\int_{H} \frac{g\left(y^{-1} x \xi\right)}{\rho(x \xi)} d \xi \\
& =T\left(L_{y} g\right)(x H) \\
& =L_{y} T g(x H) \\
& =L_{y} \eta(x H),
\end{aligned}
$$

where in the above equalities we have used the fact that the restriction $\left.\tilde{T}\right|_{L^{1}(G)}$ to $L^{1}(G)$ equals $T$ and $\tilde{T}$ is a homomorphism. Thus (2.3) becomes

$$
\left\langle\gamma \cdot \hat{\delta}_{y H}, \eta\right\rangle=\int_{G / H} \gamma(x H) L_{y} \eta(x H) d \mu(x H) .
$$

Comparing (2.2) and (2.4), we conclude (2.1).

The following lemma is a generalisation of [6, Lemma 3] to the setting of $G / H$ (see also [3, Lemma 2.1]).

Lemma 2.3. Let $G$ be a locally compact noncompact group and $H$ be a compact subgroup of $G$. Then there exist a family of compact subsets $\left(K_{\alpha}\right)_{\alpha \in I}$ of $G / H$, indexed by I, and a family $\left(y_{\alpha}\right)_{\alpha \in I} \subseteq G$ such that $K_{\alpha}^{\circ} \neq \emptyset, \bigcup_{\alpha \in I} K_{\alpha}^{\circ}=G / H,\left(K_{\alpha}\right)_{\alpha \in I}$ is closed under finite unions and $\left(y_{\alpha} K_{\alpha}\right)_{\alpha \in I}$ are pairwise disjoint.

Proof. Let $\left(K_{\alpha}\right)_{\alpha \in I}$ be a family of compact subsets with $K_{\alpha}^{\circ} \neq \emptyset$ such that $\bigcup_{\alpha \in I} K_{\alpha}^{\circ}=$ $G / H$, and assume that $I$ has minimal cardinality among all such families. By taking finite unions of such sets we may assume that $\left(K_{\alpha}\right)_{\alpha \in I}$ is closed under finite unions. Consider compact subsets $E_{\alpha}$ in $G$ so that $K_{\alpha}=q\left(E_{\alpha}\right)$, where $q$ is the canonical 
quotient map (see [1, Lemma 2.46]). Also assume that $I$ is well ordered in such a way that each nontrivial order segment $\{i \in I, i \leq j\}, j \in I$, of $I$ has smaller cardinality than $I$. We proceed by transfinite induction. Assume that for $\gamma<\alpha, \eta_{\gamma}$ is chosen. Then for any $\gamma<\alpha, \eta_{\gamma} q\left(E_{\gamma}\right) q\left(E_{\alpha}^{-1}\right)$ is compact, but by minimality of $I$, the union of these sets does not cover $G / H$. So we can choose $\eta_{\alpha} \in G / H-\bigcup_{\gamma \in I} \eta_{\gamma} q\left(E_{\gamma}\right) q\left(E_{\alpha}^{-1}\right)$. Thus for each $\gamma<\alpha$, we have $\eta_{\alpha} \notin \eta_{\gamma} q\left(E_{\gamma}\right) q\left(E_{\alpha}^{-1}\right)$. That is, $\eta_{\alpha} q\left(E_{\alpha}\right) \cap \eta_{\gamma} q\left(E_{\gamma}\right)=\emptyset$. Now choose representatives $y_{\alpha}$ of the cosets $\eta_{\alpha}$ such that $\left(y_{\alpha} K_{\alpha}\right)_{\alpha \in I}$ are pairwise disjoint.

In the following proposition (with a proof similar to that for $L^{1}(G)$ [7]) we show that $L^{1}(G / H)^{*}$ has the property $\left(F_{K}\right)$, where $\kappa$ is the least cardinality of a covering of $G / H$ by compact subsets, which is, due to compactness of $H$, equal to the compact covering number $\kappa(G)$ of $G$.

Proposition 2.4. Let $G$ be a locally compact noncompact group, $H$ be a compact subgroup of $G$ and $\kappa(=\kappa(G))$ be the least cardinality of a covering of $G / H$ by compact subsets. Then $L^{1}(G / H)^{*}$ has the property $\left(F_{\kappa}\right)$.

Proof. Let $\kappa$ be the least cardinality of a covering of $G / H$ by compact subsets and write $\left(K_{\alpha}\right)_{\alpha \in I}$ for the corresponding family of compact sets. Set

$$
\tilde{I}=I \times I, \tilde{\alpha}=(\alpha, i) \in \tilde{I}, K_{\tilde{\alpha}}=K_{(\alpha, i)}:=K_{\alpha} .
$$

Then $\left(K_{\tilde{\alpha}}\right)_{\tilde{\alpha} \in \tilde{I}}$ is a covering of $G / H$ with the same properties as the original one. Let $\left(y_{\tilde{\alpha}} K_{\tilde{\alpha}}\right)$ be as in Lemma 2.3, that is,

$$
\left(y_{\tilde{\alpha}} K_{\tilde{\alpha}}\right) \cap\left(y_{\tilde{\beta}} K_{\tilde{\beta}}\right)=\emptyset, \quad \tilde{\alpha} \neq \tilde{\beta} \in \tilde{I} .
$$

Define a partial ordering on $\tilde{I}$ by setting, for $(\alpha, i),(\beta, j) \in \tilde{I}$,

$$
(\alpha, i) \leq(\beta, j) \Longleftrightarrow K_{(\alpha, i)} \subseteq K_{(\beta, j)},
$$

and on $I$ by

$$
\alpha \leq \beta \Longleftrightarrow K_{\alpha} \subseteq K_{\beta}
$$

Define

$$
\dot{\psi}_{j}:=w^{*}-\lim _{\beta} \hat{\delta}_{y_{(\beta, j) H}}
$$

and let $\psi_{j}$ be an arbitrary Hahn-Banach extension of $\dot{\psi}_{j}$ to $L^{\infty}(G / H)^{*}$. Let $\left(\eta_{i}\right)_{i \in I} \subseteq$ $\operatorname{Ball}\left(L^{1}(G / H)^{*}\right)$. Put

$$
\eta:=\sum_{(\alpha, i) \in I \times I} L_{y_{(\alpha, i)}}\left(\chi_{K_{(\alpha, i)}} \eta_{i}\right) \frac{\rho(e)}{\rho\left(y_{(\alpha, i)}\right)} .
$$

Using Lemma 2.2, for $(\alpha, i),(\beta, j),(\gamma, k) \in I \times I$, where $(\gamma, k) \leq(\beta, j)$,

$$
\begin{aligned}
\eta \cdot \psi_{j} & =w^{*}-\lim _{\beta} \sum_{(\alpha, i) \in I \times I} \frac{\rho(e)}{\rho\left(y_{(\alpha, i)}\right)} L_{y_{(\alpha, i)}}\left(\chi_{K_{(\alpha, i)}} \eta_{i}\right) \cdot \hat{\delta}_{y_{(\beta, j) H}} \\
& =w^{*}-\lim _{\beta} \sum_{(\alpha, i) \in I \times I} \frac{\rho(e)}{\rho\left(y_{(\alpha, i)}\right)} \frac{\rho\left(y_{(\beta, j)}\right)}{\rho(e)} L_{y_{(\beta, j)}^{-1}} L_{y_{(\alpha, i)}}\left(\chi_{K_{(\alpha, i)}} \eta_{i}\right) .
\end{aligned}
$$


Using (2.5),

$$
\begin{aligned}
& \frac{\rho\left(y_{(\beta, i)}\right)}{\rho\left(y_{(\alpha, i)}\right)} \chi_{K_{(\gamma, k)}} L_{y_{(\beta, j)}^{-1}} L_{y_{(\alpha, i)}}\left(\chi_{K_{(\alpha, i)}} \eta_{i}\right) \\
& =\frac{\rho\left(y_{(\beta, j)}\right)}{\rho\left(y_{(\alpha, i)}\right)} \chi_{K_{(\gamma, k)}} \chi_{K_{(\beta, j)}} L_{y_{(\beta, j)}^{-1}} L_{y_{(\alpha, i)}}\left(\chi_{K_{(\alpha, i)}} \eta_{i}\right) \\
& =\frac{\rho\left(y_{(\beta, j)}\right)}{\rho\left(y_{(\alpha, i)}\right)} \chi_{K_{(\gamma, k)}}\left[L_{y_{(\beta, j)}^{-1}(}\left(L_{y_{(\beta, j)}} \chi_{K_{(\beta, j)}}\right)\left(L_{y_{(\alpha, i)}}\left(\chi_{K_{(\alpha, i)}} \eta_{i}\right)\right)\right] \\
& =\frac{\rho\left(y_{(\beta, j)}\right)}{\rho\left(y_{(\alpha, i)}\right)} \delta_{(\alpha, i)(\beta, j)} \chi_{K_{(\gamma, k)}} \eta_{j} .
\end{aligned}
$$

Now (2.6) and (2.7) imply that, for $j \in I$ and $(\gamma, k) \in I \times I$,

$$
\begin{aligned}
\chi_{K_{(\gamma, k)}}\left(\eta \cdot \psi_{j}\right) & =w^{*}-\lim _{\beta} \sum_{(\alpha, i) \in I \times I} \frac{\rho\left(y_{(\beta, j)}\right)}{\rho\left(y_{(\alpha, i)}\right)} \delta_{(\alpha, i)(\beta, j)} \chi_{K_{(\gamma, k)}} \eta_{j} \\
& =\chi_{K_{(\gamma, k)}} \eta_{j},
\end{aligned}
$$

which completes the proof.

Finally, we discuss the Mazur property of $L^{1}(G / H)$. Let $G$ be a locally compact noncompact group, for which $k(G) \geq 2^{b(G)}$. Let $H$ be a compact subgroup of $G$. Then $M(G)$ has the Mazur property of level $k(G)$ (see [8]). Since $L^{1}(G / H)$ is an ideal of $M(G / H)$, and $M(G / H)$ is a linear subspace of $M(G)[10], L^{1}(G / H)$ is a linear subspace of $M(G)$. Thus by [2, Remark 1.5] we conclude that $M(G / H)$ has the Mazur property of level $k(G)$.

Now Theorem 2.1 is a consequence of the above argument on the Mazur property of $L^{1}(G / H)$ together with Proposition 2.4 and Theorem 1.1.

\section{References}

[1] G. B. Folland, A Course in Abstract Harmonic Analysis (CRC Press, Boca Raton, FL, 1995).

[2] Z. Hu and M. Neufang, 'Decomposability of von Neumann algebras and the Mazur property of higher level', Canad. J. Math. 58(4) (2006), 768-795.

[3] R. A. Kamyabi-Gol, 'The topological centre of $L^{1}(K)^{* *}$, Sci. Math. Jpn. 62(1) (2005), 81-89.

[4] R. A. Kamyabi-Gol and N. Tavallaei, 'Convolution and homogeneous spaces', Bull. Iranian Math. Soc. 35(1) (2009), 129-146.

[5] A. T. Lau, 'Continuity of Arens multiplication on the dual space of bounded uniformly continuous functions on locally compact groups and topological semigroups', Math. Proc. Cambridge Philos. Soc. 99 (1986), 273-283.

[6] A. T. Lau and V. Losert, 'On the second conjugate algebra of $L^{1}(G)$ of a locally compact group', J. Lond. Math. Soc. 37 (1988), 445-460.

[7] M. Neufang, 'A unified approach to the topological centre problem for certain Banach algebras arising in abstract harmonic analysis', Arch. Math. 82(2) (2004), 164-171.

[8] M. Neufang, 'On a conjecture by Ghahramani-Lau and related problems concerning topological centres', J. Funct. Anal. 224 (2005), 217-229. 
[9] M. Neufang, 'Solution to a conjecture by Hofmeier-Wittstock', J. Funct. Anal. 217(1) (2004), $171-180$.

[10] H. Reiter and J. D. Stegeman, Classical Harmonic Analysis, 2nd edn (Oxford University Press, New York, 2000).

R. RAISI TOUSI, Department of Pure Mathematics,

Ferdowsi University of Mashhad, PO Box 1159, Mashhad 91775, Iran

e-mail: raisi@um.ac.ir

R. A. KAMYABI-GOL, Department of Pure Mathematics,

Ferdowsi University of Mashhad, PO Box 1159, Mashhad 91775, Iran and

Center of Excellence in Analysis on Algebraic Structures (CEAAS),

PO Box 1159-91775, Mashhad, Iran

e-mail: kamyabi@um.ac.ir

H. R. EBRAHIMI VISHKI, Department of Pure Mathematics,

Ferdowsi University of Mashhad, PO Box 1159, Mashhad 91775, Iran and

Center of Excellence in Analysis on Algebraic Structures (CEAAS),

PO Box 1159-91775, Mashhad, Iran

e-mail: vishki@um.ac.ir 